


\author{
L'impact de l'immigration sur les natifs des pays \\ d'accueil est une question largement débattue. \\ En théorie, le bien-être des natifs peut être affecté \\ par plusieurs canaux : la préférence ou l'aversion \\ pour la diversité culturelle, la criminalité, l'effet \\ fiscal (résultant des taxes payées et transferts reçus \\ par les immigrés), les profits des entreprises et la \\ rentabilité du capital, la structure de la demande pour \\ les biens de consommation, le capital humain moyen \\ des travailleurs et la compétitivité (qualifications \\ moyennes, innovation, adoption technologique), et les \\ effets sur le marché du travail. \\ Dans la pratique, c'est un peu plus compliqué.
}

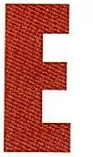

n particulier, les partis populistes européens soutiennent que l'immigration européenne est majoritairement peu qualifiée, renforce la concurrence sur le marché du travail, alourdit les déficits publics et engendre une criminalité accrue. Il n’est donc pas étonnant de constater que la perception populaire reflète ces craintes. Les sondages d'opinion publiés dans «Transatlantic Trends of Immigration » montrent que $31 \%$ des citoyens européens pensent que l'immigration est responsable de l'augmentation du chômage, et $42 \%$ pensent que l'immigration réduit leurs perspectives salariales. Les sentiments anti-immigration sont particulièrement élevés en périodes de crises. Ces mêmes sondages montrent également que les Européens estiment à $24 \%$ la part de la population immigrée dans la population totale (contre $35 \%$ aux États-Unis et $37 \%$ au Canada). La part réelle n’avoisine que $10 \%$ en Europe (14\% aux États-Unis et $20 \%$ au Canada). Les citoyens surestiment donc fortement la taille de la population immigrée. Il est donc plausible que les citoyens (et les partis populistes) aient une vision erronée des effets de l'immigration sur le bien-être.

En tant qu'économistes, nous nous concentrons sur les deux principaux effets économiques de l'immigration (marché du travail et finances publiques) et dressons un bilan de la littérature de ces quinze dernières années sur ce thème. Les effets de l'immigration légale ont été abondamment étudiés aux États-Unis, un pays aux caractéristiques très différentes des économies européennes. Plusieurs études récentes concernent des pays européens comme la France, l'Allemagne, le Royaume-Uni ou l'Espagne. La majorité de ces études aboutit à des effets quantitativement faibles et... positifs de l'immigration en matières d'emploi, de salaire et de finances publiques.

\section{L'effet sur le marché du travail}

Choc d'offre sur le marché du travail, l'immigration est susceptible d'affecter salaire et emploi des autochtones. Considérons que le stock de capital demeure inchangé à très court terme. Un accroissement de l'offre de travail (ou «demande d'emploi») devrait alors provoquer une baisse du niveau des salaires, là où ceux-ci sont flexibles, et une baisse du taux d'emploi des résidents, là où les salaires sont rigides.

À moyen terme, des changements de la taille de la population active ont de nombreux effets induits sur le marché du travail (augmentation de la demande de biens et services, des investissements, de la demande de travail, du travail au noir, ou changements dans la formation des salaires). En particulier, lorsque les immigrés consomment, épargnent et investissent, et/ou lorsque des investissements étrangers répondent à l'augmentation du rendement du capital, l'emploi augmente.

Enfin, une nouvelle vague d'études récentes démontre que les immigrants ont des caractéristiques différentes des résidents (qualifications, structure par âge, spécialisations différentes). S'il est 
possible que l'immigration engendre une concurrence plus forte pour nos travailleurs jeunes et non qualifiés, il est également vraisemblable que les immigrés occupent des emplois que les natifs ne désirent pas ou pour lesquels l'offre de travail des natifs est déficitaire. Il y aurait donc « complémentarité», plutôt que «substituabilité », entre le travail des natifs et le travail des immigrés.

Que dit la littérature empirique? Une vaste littérature porte sur l'effet de l'immigration sur les salaires des résidents. Partout dans le monde, la corrélation spatiale entre les salaires et le nombre d'immigrés est extrêmement faible. En particulier, les études américaines et européennes concluent à des taux de corrélation positifs mais négligeables : les salaires sont légèrement supérieurs dans les villes ou régions où l'immigration est plus importante. Les détracteurs souligneront que cette corrélation positive reflète une causalité inverse (les immigrés se localisent là où les salaires sont élevés) ou une mobilité des natifs (les natifs fuient la concurrence engendrée par l'immigration en changeant de lieu de travail et/ou de résidence). Pour ces raisons, des économistes réputés tels que George Borjas préconisent de mener des études au niveau spatial le plus large («agrégé ») possible.

Les études menées au niveau agrégé offrent des résultats différents. Les partisans de la substituabilité sont de plus en plus minoritaires. Les travaux de George Borjas sont considérés comme les plus pessimistes. Ils montrent que l'immigration aux États-Unis exerce un effet légèrement négatif sur le salaire moyen des citoyens américains (entre un dixième et un demi \%) ${ }^{1}$. Néanmoins, la plupart des travaux récents (en particulier ceux menés sur la France, le Royaume-Uni ou l'Allemagne) montrent que la complémentarité entre natifs et immigrés est importante. Les résultats les plus optimistes sont pré-

\section{LE CLICHÉ SELON LEQUEL LES IMMIGRÉS VONT OCCUPER LES EMPLOIS DES POPULATIONS RÉSIDENTES NE RÉSISTE PAS AUX ÉTUDES SCIENTIFIQUES EXISTANTES. CELUI QUI VOIT DANS L'IMMIGRÉ UNE SANGSUE VIDANT LES CAISSES DE LA SÉCURITÉ SOCIALE SE TROMPE MANIFESTEMENT.}

sentés par David Card pour les États-Unis ou Marco Manacorda, Alan Manning et Jonathan Wadsworth pour l'Europe ${ }^{2}$. L'immigration apparaît de plus en plus comme un facteur permettant de «huiler» le marché du travail et d'augmenter la compétitivité des entreprises, la productivité et la demande pour le travail des natifs. L'immigration serait donc bénéfique pour l'emploi et le salaire des natifs. Elle n'aurait des effets significativement négatifs que pour les cohortes antérieures d'immigrés.

\section{L'effet sur les finances publiques}

Les immigrants non qualifiés sont souvent perçus comme des consommateurs importants de services publics et transferts sociaux. Ceci est particulièrement vrai si les immigrants choisissent leur destination sur base de la générosité des systèmes de protection sociale. L'effet de l'immigration sur les finances publiques dépend donc de la générosité des transferts ainsi que du niveau de qualification et de la structure par âge des immigrés. Pour cerner cet impact, il est utile de calculer les taxes nettes payées par les immigrés, c'est-à-dire la différence entre les taxes payées à l'État et les transferts reçus de l'État sur une année.

Ces profils de taxes nettes ont été calculés dans différents pays. Ces études de finances publiques révèlent des résultats plutôt optimistes. Des analyses menées aux États-Unis, en Allemagne ou en Espagne montrent que l'immigration induit une diminution de la pression budgétaire sur les résidents. Le cas espagnol est par ticulièrement intéressant dans la mesur où l'immigration y est majoritairement no qualifiée. L'effet bénéfique est lié au rajeu nissement permanent de la population per mis par l'immigration. Dans le contexte d vieillissement de population, une augmen tation de l'immigration est jugée comm désirable du point de vue des finances pu bliques. Ce résultat a été confirmé pour plupart des économies européennes dan une étude récente de la Banque mondiale basée sur les données individuelles de l'en quête SILC européenne (Study of Incom and Living Conditions) 3 .

Une étude française distingue les ni veaux de qualification des immigrés 4 . Elle part d'une désagrégation des taxes nette des immigrés selon trois niveaux de qua lification (les immigrés avec diplôme se condaire supérieur, ceux avec diplôme post-secondaire et les moins qualifiés san diplôme ou avec diplôme inférieur au se condaire supérieur). L'effet varie forte ment avec le niveau d'éducation. Les im migrés très qualifiés (diplôme supérieu au secondaire) contribuent davantage que l'autochtone moyen aux recettes nettes de l'État. La contribution des immigrés moyennement qualifiés (diplôme secondaire) est similaire à celle des résidents La contribution des immigrés peu qualifiés (diplôme inférieur au secondaire) est modeste, mais positive entre 25 et 55 ans. À long terme (sur l'ensemble de leur vie), ces immigrés non qualifiés coûtent plus cher 
que ce qu'ils ne rapportent aux finances publiques. À court terme, lorsqu'ils sont jeunes, ils contribuent faiblement mais positivement aux finances de l'État.

\section{Des arguments idéologiques}

Les études qui ont analysé ensemble les différents effets concluent que l'effet favorable sur les finances publiques et l'emploi impliquent que toutes les générations et toutes les catégories de résidents ont modestement bénéficié de l'afflux migratoire (aux États-Unis et en France) ${ }^{5}$. Le seul véritable groupe à risque est celui des populations résidentes jeunes et moins qualifiées. Ce sont elles qui pourraient, dans les scénarios les plus pessimistes, subir des conséquences négatives sur le marché du travail.

Dans ce contexte, nombre d'opinions anti-immigration apparaissent guidées par des arguments essentiellement idéologiques et des clichés peu robustes, voire profondément inexacts. Le cliché selon le-

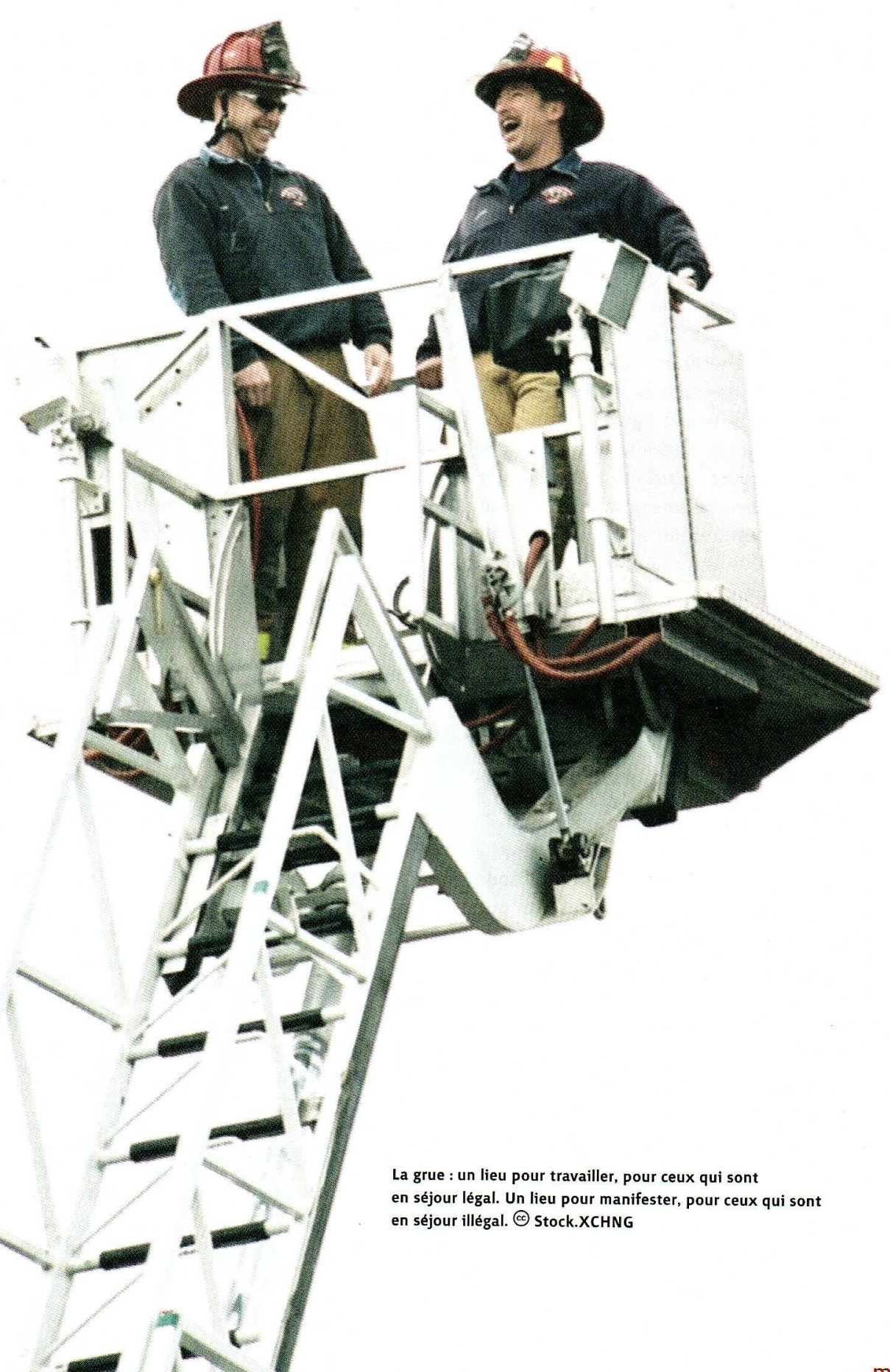

quel les immigrés vont occuper les emplois des populations résidentes ne résiste pas aux études scientifiques existantes. Celui qui voit dans l'immigré une sangsue vidant les caisses de la sécurité sociale se trompe manifestement.

Quid des arguments non économiques? Le lien entre immigration et criminalité est plus complexe. Certaines études trouvent un effet positif, fortement dépendant des pays d'origine et régions de destination des immigrés ${ }^{6}$. D'autres études mentionnent que l'impact sur la criminalité n'est perceptible qu'à partir de la seconde génération, et dépend de l'intégration économique et sociale de la première génération ${ }^{7}$. Nous ne pouvons non plus nier le fait que les citoyens européens ont des attitudes diverses par rapport à la diversité culturelle. En particulier, les sondages d'opinion révèlent clairement une crainte de l'islam ( $42 \%$ des Français ont peur de l'islam, selon un sondage réalisé par l'IFOP pour Le Monde). Toutefois, l'utilisation de prétextes économiques pour freiner l'immigration n'a guère de fondement scientifique.

1 Voir Borjas, G. (2003), "The Labor Demand Curve is Downward Sloping: Reexamining the Impact of Immigration on the Labor Market", Quarterly Journal of Economics 118, no. 4: 1335-1374. Ou plus récemment, Borjas, G. \& J. Grogger \& G. Hanson (2008), "Imperfect Substitution between Immigrants and Natives: A Reappraisal”, NBER Working Paper \# 13887, Cambridge, MA.

2 Voir Card, D. (2009), “Immigration and inequality", American Economic Review 99 (2): 1-21. Voir aussi Manacorda M. \& A. Manning \& J. Wadsworth (à paraitre), "The Impact of Immigration on the Structure of Wages: Theory and Evidence from Britain", Journal of the European Economic Association.

3 Voir Barbone, L. \& M. Bontch-Osmolovsky \& S. Zaidi (2009), "The foreign-born population in the European Union and its contribution to national tax and benefit systems: some insights from recent household survey data", Policy Research Working Paper Series 4899, The World Bank.

4 Voir Chojnicki, X. (2006), « Vieillissement démographique et immigration: un modèle de comptabilité générationnelle appliqué à la France », Économie et Prévisions 174 (3), 39-57.

5 Voir Chojnicki, X. \& F. Docquier \& L. Ragot (2007), "Should the US have locked heaven's door? Reassessing the benefits of postwar immigration", Journal of Population Economics (2011), 24, 317-359.

6 Voir Bell, B. \& S. Machin \& F. Fasani (2010), “Crime and Immigration: Evidence from Large Immigrant Waves", CReAM Discussion Paper Series 1012, Centre for Research and Analysis of Migration (CReAM), Department of Economics, University College London.

7 Voir Mariani, F. (2011), "Exporting criminals?", Mimeo, IRES - Université Catholique de Louvain. 